\title{
Relationship of cytokines and cytokine signaling to immunodeficiency disorders in the mouse
}

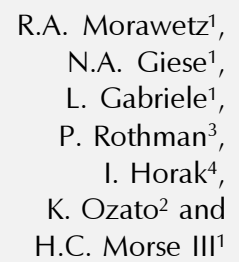

\author{
${ }^{1}$ Laboratory of Immunopathology, National Institute of Allergy and Infectious \\ Diseases, and ${ }^{2}$ Laboratory of Molecular Growth Regulation, National Institute of Child \\ Health and Human Development, National Institutes of Health, Bethesda, MD, USA \\ ${ }^{3}$ Columbia University, New York, NY, USA \\ ${ }^{4}$ Department of Molecular Genetics, Research Institute of Molecular Pharmacology, \\ Berlin, Germany
}

Correspondence

H.C. Morse III

LIP, NIAID, NIH

9000 Rockville Pike

Bethesda, MD 20892-0760

USA

Fax: 301-402-0077

Presented at the International Meeting on Cytokines, Angra dos Reis, RJ, Brasil, November 24-28, 1996.

$\ldots \ldots \ldots \ldots \ldots \ldots \ldots \ldots \ldots \ldots \ldots$

Received September 4, 1997 Accepted September 22, 1997 ......................

\section{Abstract}

The contributions of cytokines to the development and progression of disease in a mouse model of retrovirus-induced immunodeficiency (MAIDS) are controversial. Some studies have indicated an etiologic role for type 2 cytokines, while others have emphasized the importance of type 1 cytokines. We have used mice deficient in expression of IL-4, IL-10, IL-4 and IL-10, IFN- $\gamma$, or ICSBP - a transcriptional protein involved in IFN signaling - to examine their contributions to this disorder. Our results demonstrate that expression of type 2 cytokines is an epiphenomenon of infection and that IFN- $\gamma$ is a driving force in disease progression. In addition, exogenously administered IL-12 prevents many manifestations of disease while blocking retrovirus expression. Interruption of the IFN signaling pathways in $\mathrm{ICSBP}^{-/-}$ mice blocks induction of MAIDS. Predictably, ICSBP-deficient mice exhibit impaired responses to challenge with several other viruses. This immunodeficiency is associated with impaired production of IFN- $\gamma$ and IL-12. Unexpectedly, however, the ICSBP ${ }^{-/-}$mice also develop a syndrome with many similarities to chronic myelogenous leukemia in humans. The chronic phase of this disease is followed by a fatal blast crisis characterized by clonal expansions of undifferentiated cells. ICSBP is thus an important determinant of hematopoietic growth and differentiation as well as a prominent signaling molecule for IFNs.

Mice infected with the LP-BM5 mixture of murine leukemia viruses (MuLV) develop a syndrome, termed mouse AIDS (MAIDS), characterized by progressive lymphoproliferation and increasing severe immunodeficiency $(1,2)$. A replication-defective virus (BM5def) in this mixture encodes a unique Gag protein that is the critical requirement for
Key words

- ICSBP

- Immunodeficiency

- Interferon

- MAIDS disease induction $(3,4)$. Mice injected with BM5def alone develop disease without the requirement for virus spread (5), but most studies of MAIDS have been performed with mice inoculated with ecotropic and MCF helper viruses that mediate cell-to-cell spread of BM5def.

Susceptibility to induction of MAIDS fol- 
lowing infection with BM5def is strain dependent, with some mice, such as C57BL/6 (B6) and C57L, developing signs of disease within 2 to 3 weeks of infection, while others, such as A/J, 129 or RIIIS, show no signs of disease through a year or more after infection (6-8). These strain differences are genetically determined, and mapping studies have localized strong determinants of resistance to both class I and class II genes of the MHC as well as undefined genes not linked to the MHC $(6,8,9) . \mathrm{CD}^{+} \mathrm{T}$ cells contribute to disease resistance (10), but other cells also play an important role, since mice of resistant genotypes deficient in $\mathrm{CD}^{+}$cells ( $(32 \mathrm{~m}$ knockouts) or perforin (perforin knockouts) develop disease with kinetics that is markedly delayed in comparison with that of fully susceptible mice (Tang Y, Hügin AW, Giese NA, Gabriele L, Kägi D and Morse III HC, unpublished data).

In susceptible mice, the critical viral determinants of disease induction include the MA(p15) and $\mathrm{p} 12$ regions of BM5def Gag (11), which differ greatly from the same regions in nonpathogenic ecotropic MuLV. Mature B cells are the primary targets of infection by BM5def, and infection of B cells is required for efficient infection of $\mathrm{T}$ cells and macrophages $(12,13)$. Expression of BM5def in B cells and macrophages is not sufficient to induce disease, since mice carrying the nude mutation on a susceptible background express high levels of defective virus but do not develop MAIDS $(14,15)$. However, infected nudes given purified $\mathrm{CD}^{+} \mathrm{T}$ cells do develop disease, indicating a requirement for T/B cell interactions (15). These interactions appear to be antigen-specific, since disease does not develop in mice deficient in expression of MHC class II molecules (15) or CD4 (Morse III HC, unpublished observations). Costimulation would appear to be an additional requirement for development of full-blown disease, as progression of MAIDS is delayed in mice treated with blocking antibodies to CD11 $\mathrm{a}$ and CD54
(16) or deficient in expression of CD80 (B7-1; Kim Y, Giese NA, Sharpe AH and Morse III $\mathrm{HC}$, unpublished observations) or CD28 (Giese NA, Morse III HC and Abe R, unpublished observations). The CD40/CD40L interactions would also appear to be crucial, since disease is completely blocked in mice treated with antibodies to CD40L (17). In spite of these indications that MAIDS is likely to be antigen driven, the nature of the necessary determinant has not been defined.

In recent years, considerable attention has been focused on the possibility that cytokines may be another critical determinant of T/B cell interactions in MAIDS. Studies of parasitic infections advanced the paradigm that early, polar deviation of responses toward type 1 vs type 2 cytokines determined protective and nonprotective immune responses, respectively. These studies also showed that mice of susceptible strains could be rendered resistant by inhibiting development of the type 2 response or stimulating expression of type 1 cytokines.

Initial studies of supernatants of concanavalin A-stimulated spleen cells from MAIDS-susceptible B6 mice showed that at 1 week after infection there was a burst of cytokine expression with a profile that could be interpreted as mixed type 1 /type 2 or Th0 (18). A similar pattern of cytokines was expressed transiently by unstimulated spleen cells from mice infected for 1 week, but at latertimes postinfection, spontaneous expression was no longer detectable. Studies of stimulated cells from mice infected 2 weeks or more showed that expression of IL-2 and IFN- $\gamma$ decreased progressively, while expression of IL-4 and IL-10 increased in a reciprocal fashion (18). The observation that serum IgE levels increased in concert with induced IL-4 expression was taken to be an indication of IL-4 expression in vivo and thus a validation of the in vitro assays. These observations had some similarities to those in studies of peripheral blood lymphocytes of $\mathrm{HIV}^{+}$subjects, suggesting that there might be common 
mechanisms contributing to retrovirusinduced immunodeficiency in mice and humans (19). The model proposed was that type 2 cytokines were responsible for downregulation of host cell-mediated immunity governed by type 1 cytokines.

This model was tested in MAIDS-susceptible mice in three ways: first, by determining whether enforced expression of type 1 cytokines would be protective against disease, and second, by judging whether mice impaired in their ability to produce type 2 cytokines would be resistant to MAIDS. A third approach was to study MAIDS-resistant mice for their pattern of cytokine expression with the expectation that they would exhibit a type 1 profile.

The first approach involved treatment of B6 mice with IL-12 to stimulate expression of IFN- $\gamma$ and to bias T helper development toward Th1. Treatment was initiated at the time of infection or at 2 to 4 weeks after virus inoculation (20). Treatment beginning at the time of infection markedly inhibited the development of lymphoproliferation, as well as signs of B cell activation, and protected the ability of stimulated spleen cells to produce IFN- $\gamma$ and IL-2. IL-12- used as a therapeutic agent - also had strikingly beneficial effects in limiting lymphoproliferation and restoring immune function. The beneficial effects of IL-12 were dependent on the expression of IFN- $\gamma$, as infected IFN- $\gamma$ knockout mice were not protected from disease when treated with IL-12 (20). These results were in keeping with the model that type 1 cytokines would mediate a resistant phenotype in mice genetically susceptible to MAIDS.

The first reported studies of mice deficient in expression of IL-4 appeared to lend further strong support to this model (21). Progeny of $F_{2}$ mice from crosses between strain 129 IL-4-deficient mice and B6 showed that almost all $\mathrm{IL}-4^{+/+}$mice developed disease within 12 weeks of infection, whereas IL-4 ${ }^{-/-}$ mice generally developed disease after more than 20 weeks. Interpretation of this study is complicated by the facts that strain 129 mice are completely resistant to MAIDS and that the $+/+$ and $-/-$ mice used in the study were progeny of crosses between selected $+/+$ and -/- animals instead of being strictly $\mathrm{F}_{2}$ mice (Kanagawa $\mathrm{O}$, personal communication). This raises the possibility that genes determining the resistance to MAIDS of strain 129 might inadvertently have become fixed in the -/- mice.

At the time of this report, we had backcrossed an independently generated IL-4 defect from strain 129 onto a B6 background. Analyses of these -/- congenics showed them to be indistinguishable from $\mathrm{B}^{+/+}$mice for their sensitivity to MAIDS, leading us to conclude that IL-4 is not required for induction or progression of disease (22). This conclusion has since been supported by studies of yet a third IL-4 knockout strain generated, this time, using B6 ES cells. Mice of this strain, which carries no 129 genes, showed the same sensitivity to induction of MAIDS as the congenics tested previously (Morawetz RA, NobenTrauth $\mathrm{N}$ and Morse III HC, unpublished data). In addition, detailed studies of the response to infection of mice from a series of genetic crosses between B6 and 129 mice, some including mice with mutant IL-4 genes and some not, have revealed no association between IL-4 deficiency and resistance to MAIDS (Morawetz RA, Coffman RL and Morse III HC, unpublished data); however, mice homozygous for the $\mathrm{Fv}-1^{\mathrm{n}}$ allele of strain 129 were much more resistant to disease than mice carrying the $\mathrm{B} 6$ allele, $\mathrm{Fv}-1^{\mathrm{b}}$. This would be expected from the restriction to spread of B-tropic helper viruses in the LP-BM5 mixture imposed by then allele of $\mathrm{F} v$ - 1 (Morawetz RA, Coffman RL and Morse III HC, unpublished data). These findings strongly suggest that MAIDS-resistant mice in the Kanagawa study were homozygous $\mathrm{Fv}-1^{\mathrm{n}}$, in addition to being IL-4 ${ }^{-/}$, while sensitive mice were most likely $\mathrm{Fv}-1^{\mathrm{b}}$ and $\mathrm{IL}-4^{+/+}$.

During the course of these studies, it was found that both IL-4 $4^{+/+}$and IL-4/- mice with 
MAIDS had comparably high levels of serum IgE (23), even though IL-4 has long been considered to be an absolute requirement for class switching to IgE. The mechanisms involved in IgE expression are fairly well understood, consisting of induction of germline transcripts $(g \varepsilon)$ followed by switch recombination and the generation of productive transcripts $(p \varepsilon)$, which are translated to yield $\operatorname{IgE}$ protein. Induction of ge following IL-4 engagement of its receptor is thought to occur by activation of JAK1 and JAK3, leading to phosphorylation and activation of STAT6, which enters the nucleus and binds to $\gamma$-activated sequences (GAS) in the IgE heavy chain promoter. STAT6 would appear crucial to IgE switching, since STAT6 knockout mice have no serum IgE (e.g., Ref. 24).

The observation that IL-4 is not required for IgE expression in mice with MAIDS suggests that the MAIDS-defective virus may substitute for some protein in the IL-4 signaling pathway, serving to activate JAK/STAT signaling or perhaps acting as a surrogate transcription factor. As an initial approach to understanding IL-4-independent IgE induction in mice with MAIDS, we have studied B cell lymphomas expressing BM5def Gag (Morawetz RA, Rothman P and Morse III $\mathrm{HC}$, unpublished data). Some of these lymphomas were obtained from mice with MAIDS (25), while others were non-MAIDS lymphomas infected with BM5def. Remarkably, several MAIDS lymphomas expressed $\mathrm{g} \varepsilon$ and $\mathrm{p} \varepsilon$ transcripts. In addition, CH12.LX cells infected with BM5def expressed substantially increased levels of ge transcripts, again suggesting that the Gag protein is involved in signaling to the nucleus. None of the lines constitutively expressed activated STAT6, however, even though all were inducible for STAT6 when treated with IL-4. This strongly suggests that the Gag protein does not affect activation of JAK1 and JAK3, which would lead to activation of STAT6. The possibility that the virus might affect expression of IL-13 - which can signal IgE switch recombination in human cells - seems unlikely; B cells from normal or IL-4/-- mice could not be induced to switch to IgE following stimulation with high doses of IL-13 in vitro (Coffman RL, personal communication). While the mechanisms governing IL-4-independent induction of IgE thus remain poorly understood, the initial interpretation of the basis for IgE expression in MAIDS was clearly wrong. Studies are in progress to further define this phenomenon.

The demonstration that IL-4 is not required for normal development of MAIDS left open the possibility that other type 2 cytokines could be required for induction and progression of this disease. Strong evidence against this possibility was generated in studies of IL-10 knockouts and IL-4/IL-10 double knockouts on a B6 background. Both strains of mutant mice developed lymphoproliferation and immunodeficiency with kinetics nearly identical to those of wild-type mice (Morawetz RA, Rajewsky K, Kuhn R, Muller W and Morse III HC, unpublished observations). In addition, MAIDS-susceptible BALB.B mice carrying an IL-6 knockout were normally susceptible to induction of MAIDS (McCarty TC and Morse III HC, unpublished observations). These findings demonstrated that expression of type 2 cytokines in MAIDS was an epiphenomenon of infection rather than a driving force for development of disease.

During the course of these studies, the question of cytokine expression during the induction and progression of MAIDS was readdressed using semiquantitative RT-PCR to evaluate cytokine transcripts. These studies showed that in spleens of infected B6 mice, the levels of transcripts for IL-2, IL-4, IL-10, IL-12, IFN- $\gamma$, and TNF- $\alpha$ were all significantly increased at 1 week after infection and that transcripts for all cytokines, except IL-2, continued to increase 4 months after infection (26). PCR analyses of mRNA in spleens of the MAIDS-resistant strain BALB/c showed a pattern of cytokine expression simi- 
lar to that of B6 at 1 week postinfection, but after that, the levels of transcripts for all cytokines returned rapidly to preinfection levels. Since it was known that the type 2 cytokines contributed little, if anything, to the development of MAIDS, we examined the possible contributions of IFN- $\gamma$ expression to disease sensitivity or resistance in studies of infected B6 and BALB/c IFN- $\gamma$ knockouts, respectively (26). The B6 IFN- $\gamma^{-/-}$mice developed MAIDS but with a delayed time course for progression of lymphoproliferation, indicating that IFN- $\gamma$ was needed for characteristic disease progression. This conclusion is strongly supported by the observation that $\mathrm{B} 6$ mice treated with neutralizing $\mathrm{mAb}$ to IFN- $\gamma$ were protected against lymphoproliferation and immunodeficiency (27). IFN- $\gamma$ was found to play no role in resistance to MAIDS, however, since the BALB $/ \mathrm{c} \mathrm{IFN-} \gamma^{-/-}$mice showed no signs of disease at any time after infection.

Taken together, the studies of B6 mice treated with IL-12 and B6 IFN- $\gamma$ knockouts showed that high-level expression of this cytokine could protect against disease, while low-level expression contributed to disease progression. The potential contributions of type I IFNs (IFN- $\alpha / \beta)$ to MAIDS sensitivity are controversial. Early studies of serum IFN showed that mice infected for several weeks were unable to produce IFN- $\alpha / \beta$ after in vivo challenge with Newcastle disease virus (28). Subsequent RT-PCR analyses of IFN- $\alpha / \beta$ transcripts in spleens of infected mice showed a slow, low-level accumulation of transcripts over time in B6 mice and transient, modest levels of expression in BALB/c and 129 mice. MAIDS-resistant mice treated with neutralizing antibodies to IFN- $\alpha / \beta$ were unchanged in their response to infection (Giese NA, Morawetz RA, Gresser I and Gabriele L, unpublished observations). This led us to conclude that expression of type I IFNs is induced by infection but is not of importance to resistance to disease.

More recently, others have indicated that an IFN- $\alpha / \beta$ response is generated within hours of infection of MAIDS-resistant but not MAIDS-sensitive mice (28). They also showed that sensitive mice stimulated to express high levels of IFN- $\alpha / \beta$ at the time of infection exhibited reduced lymphoproliferation and less pronounced immune defects when compared with untreated infected mice (28). The interpretation was that type I IFNs are important to disease resistance. This view was inconsistent with our findings but, together with the documented importance of type I IFN in MAIDS (29), prompted us to investigate pathways of type I and type II IFN signaling in infected mice.

Both types of IFN signal to the nucleus by activation of different JAK/STAT pathways (reviewed in Ref. 30). Engagement of type I IFN receptors induces activation of JAK 1 and Tyk2, which phosphorylate STAT1a, STAT1b, and STAT2. The STATs form heterodimers that associate with p48 (ISGF3 $\gamma$ ) to form a complex, ISGF3, that has DNA-binding specificity for sequences designated IFN- stimulated response elements (ISRE). Binding of the complex to ISRE results in transcriptional induction of specific genes that confer cellular responses to type IIFNs. Binding of IFN- $\gamma$ to its receptor stimulates activation of JAK1 and JAK3, which activate STAT1. Homodimers of STAT1 are transported to the nucleus, where they bind to DNA sequences, designated IFN- $\gamma$ activation sites (GAS), in the promoters of IFN type II-responsive genes.

These two signaling systems were once thought to be independent, but it is now recognized that there is considerable crosstalk between them. For example, IFN type II response factors (IRF) besides ISGF3 $\gamma$ have specificity for ISRE. One IRF, designated interferon consensus sequence binding protein (ICSBP), has GAS sequences in its promoter and its expression is regulated by IFN- $\gamma$ (31).

Using electrophoretic mobility shift assays (EMSA) of different IRF family members in nuclear extracts prepared from spleens of mice with MAIDS, it was found that 
bands associated with binding of IRF-2 or of complexes formed between IRF-2 and ICSBP were lost within a few weeks after infection (Giese NA, Ozato K, Nelson N and Morse III $\mathrm{HC}$, unpublished data). The loss of these binding specificities was unique to infection with BM5def since it was not observed in mice infected with nonpathogenic ecotropic virus. $T$ cells were critical for this effect as it was not seen in nude mice infected with the LP-BM5 virus mixture. The effect extended to some transcription factors not involved in IFN signaling; YY1 binding was reduced in EMSA. In contrast, other transcription factors such as NFKB were normal (31). Of considerable interest was the finding that, while STAT1 activity was upregulated in MAIDS-resistant mice infected with the virus mixture as well as in sensitive mice infected with ecotropic virus only, it was reduced in MAIDS-sensitive mice.

During the course of these studies, our laboratories were involved in analyses of mice carrying a mutant ICSBP gene generated by gene knockout technology. These studies showed the mice to have two distinct phenotypes, enhanced susceptibility to several virus infections associated with reduced expression of IFN- $\gamma$ by non-T cells, and an unusual dysregulation of hematopoiesis (32). The latter syndrome resembled chronic myelogenous leukemia in humans in several respects. The parallels included a prolonged phase of neutrophilia followed by a malig- nant blast cell crisis that was clonal and transferable to normal mice (32).

By EMSA, IRF family binding activities to ISRE by nuclear extracts from spleens of $\mathrm{ICSBP}^{-/-}$mutant mice resembled, in some ways, those seen in mice with MAIDS. ICSBP activity was absent as expected but IRF-2 binding was undetectable, while NFKB binding to a control probe was normal (32). To determine whether signaling pathways regulated by ICSBP were important for the development of MAIDS, ICSBP ${ }^{-/-}$mice were infected with LP-BM5 viruses. Infection induced striking splenomegaly that, by histopathology, resembled exacerbated chronic myelogenous leukemia with no signs of MAIDS (Giese NA, Masumi A, Ozato K and Morse III $\mathrm{HC}$, unpublished observations). The means by which retroviruses convert a chronic myeloid disease into an acute one are not known but are under study.

Based on this study, MAIDS can be seen as a disease of disordered extracellular and intracellular signaling. Abnormalities induced in B cells by infection with BM5defare not expressed to any great extent without signals provided by $\mathrm{T}$ cells. At least some of these abnormalities can be attributed to altered expression of IRF family members and changes in JAK/STAT expression. These observations indicate that development of MAIDS is dependent on modulation of cellular functions regulated by genes with ISRE or GAS sequences in their promoters.

\section{References}

1. Morse III HC, Chattopadhyay SK, Makino M, Fredrickson TN, Hügin AW \& Hartley JW (1992). Retrovirus-induced immunodeficiency in the mouse: MAIDS as a model for AIDS. AIDS, 6: 607-621 (Editorial).

2. Morse III HC, Giese N, Morawetz R, Tang Y, Gazzinelli R, Kim W-K, Chattopadhyay S \& Hartley JW (1995). Cells and cytokines in the pathogenesis of MAIDS, a retrovirus-induced immunodeficiency syndrome of mice. Springer Seminars in Immunopathology, 17: 231-245.
3. Jolicoeur P (1991). Murine acquired immunodeficiency syndrome (MAIDS): an animal model to study the AIDS pathogenesis. FASEB Journal, 5: 2398-2405.

4. Chattopadhyay SK, Sengupta DN, Fredrickson TN, Morse III HC \& Hartley JW (1991). Characteristics and contributions of defective, ecotropic, and mink cell focus-inducing viruses involved in a retrovirus-induced immunodeficiency syndrome of mice. Journal of Virology, 65: 4232-4241.
5. Aziz DC, Hanna Z \& Jolicoeur P (1989). Severe immunodeficiency disease induced by a defective murine leukemia virus. Nature, 338: 505-508.

6. Huang $M$, Simard C \& Jolicoeur P (1989). Immunodeficiency and clonal growth of target cells induced by helper-free defective retrovirus. Science, 246: 1614-1616.

7. Makino M, Morse III HC, Fredrickson TN \& Hartley JW (1990). H-2-associated and background genes influence the development of a murine retrovirus-induced immunodeficiency syndrome. Journal of Immunology, 144: 4347-4355. 
8. Makino M, Davidson WF, Fredrickson TN, Hartley JW \& Morse III HC (1991). Effects of non-MHC loci on resistance to retrovirus-induced immunodeficiency in mice. Immunogenetics, 33: 345-351.

9. Makino $M$, Tang $Y$, Murphy DB, Fredrickson TN, Okada Y, Fujiwara M, Chattopadhyay SK, Mizuochi T, Komuro K, Morse III HC \& Hartley JW (1994). Influence of $\mathrm{H}-2$ class II antigens on the development of murine AIDS. Journal of Immunology, 152: 4157-4164.

10. Makino M, Chattopadhyay SK, Hartley JW \& Morse III HC (1992). Analysis of role of $\mathrm{CD}^{+}+\mathrm{T}$ cells in resistance to murine AIDS in A/J mice. Journal of Immunology, 149: 1702-1706.

11. Kubo Y, Kakimi K, Higo K, Wang L, Kobayashi H, Kuribayashi K, Masuda T, Hirama T \& Ishimoto A (1994). The p15gag and $p 12$ gag regions are both necessary for the pathogenicity of the murine AIDS virus. Journal of Virology, 68: 5532-5537.

12. Huang M, Simard C, Kay DG \& Jolicoeur $P$ (1991). The majority of cells infected with the defective murine AIDS virus belong to the B-cell lineage. Journal of Virology, 65: 6562-6571.

13. Kim WK, Tang Y, Kenny JJ, Longo DL \& Morse III HC (1994). In murine AIDS, B cells are early targets of defective virus and are required for efficient infection and expression of defective virus in $T$ cells and macrophages. Journal of Virology, 68: 6767-6769.

14. Mosier DE, Yetter RA \& Morse III HC (1987). Functional T lymphocytes are required for a murine retrovirus-induced immunodeficiency disease (MAIDS). Journal of Experimental Medicine, 165: 1737-1742.

15. Giese NA, Giese T \& Morse III HC (1994). Murine AIDS is an antigen-driven disease: requirements for major histocompatibility complex class II expression and $\mathrm{CD} 4+\mathrm{T}$ cells. Journal of Virology, 68: 5819-5824.

16. Makino M, Yoshimatsu K, Azuma M, Okada Y, Hitoshi Y, Yagita H, Takatsu K \& Komuro K (1995). Rapid development of murine AIDS is dependent on signals provided by CD54 and CD11a. Journal of Immunology, 155: 974-981.
17. Green KA, Crassi KM, Laman JD, Schoneveld A, Strawbridge RR, Foy TM, Noelle RJ \& Green WR (1996). Antibody to the ligand for CD40 (gp39) inhibits murine AIDS-associated splenomegaly, hypergammaglobulinemia, and immunodeficiency in disease-susceptible C57BL/ 6 mice. Journal of Virology, 70: 25692575.

18. Gazzinelli RT, Makino M, Chattopadhyay SK, Snapper CM, Sher A, Hügin AW \& Morse III HC (1992). CD4+ subset regulation in viral infection. Preferential activation of Th2 cells during progression of retrovirus-induced immunodeficiency in mice. Journal of Immunology, 148: 182188.

19. Sher A, Gazzinelli RT, Oswald IP, Clerici M, Kullberg M, Pearce EJ, Berzofsky JA, Mosmann TR, James SL \& Morse III HC (1992). Role of T-cell derived cytokines in the downregulation of immune responses in parasitic and retroviral infection. Immunological Reviews, 127: 183-204

20. Gazzinelli RT, Giese NA \& Morse III HC (1984). In vivo treatment with interleukin 12 protects mice from immune abnormalities observed during murine acquired immunodeficiency syndrome (MAIDS). Journal of Experimental Medicine, 180: 2199-2208

21. Kanagawa O, Vaupel BA, Gayama S, Koehler G \& Kopf M (1993). Resistance of mice deficient in IL-4 to retrovirus-induced immunodeficiency syndrome (MAIDS). Science, 262: 240-242.

22. Morawetz RA, Doherty TM, Giese NA, Hartley JW, Müller W, Kühn R, Rajewsky K, Coffman R \& Morse III HC (1994). Resistance to murine acquired immunodeficiency syndrome. Science, 265: 264-267.

23. Morawetz RA, Gabriele L, Rizzo LV, Noben-Trauth N, Kühn R, Rajewsky K, Müller W, Doherty TM, Finkelman F, Coffman RL \& Morse III HC (1996). IL-4-independent immunoglobulin class switch to $\lg E$ in the mouse. Journal of Experimental Medicine, 184: 1651-1661.

24. Kaplan MH, Schindler U, Smiley ST \& Grusby MJ (1996). Stat6 is required for mediating responses to IL-4 and for the development of Th2 cells. Immunity, 4: 313-319.
25. Klinken SP, Fredrickson TN, Hartley JW Yetter RA \& Morse III HC (1988). Evolution of $B$ cell lineage lymphomas in mice with a retrovirus-induced immunodeficiency syndrome, MAIDS. Journal of Immunology, 140: 1123-1131.

26. Giese NA, Gazzinelli RT, Actor JK, Morawetz RA, Sarzotti M \& Morse III HC (1996). Retrovirus-elicited interleukin-12 and tumour necrosis factor- $\alpha$ as inducers of interferon- $\gamma$-mediated pathology in mouse AIDS. Immunology, 87: 467-474.

27. Uehara $S$, Hitoshi $Y$, Numata $F$, Makino M, Howard M, Mizuochi T \& Takatsu K (1994). An IFN- $\gamma$-dependent pathway plays a critical role in the pathogenesis of murine immunodeficiency syndrome induced by LP-BM5 murine leukemia virus. International Immunology, 6: 1937-1947.

28. Pitha PM, Biegel D, Yetter RA \& Morse III $H C$ (1988). Abnormal regulation of IFN- $\alpha$, $-\beta$, and $-\gamma$ expression in MAIDS, a murine retrovirus-induced immunodeficiency syndrome. Journal of Immunology, 141: 3611-3616.

29. Heng JKM, Price $P$, Lai CM \& Beilharz MW (1996). Alpha/beta interferons increase host resistance to murine AIDS. Journal of Virology, 70: 4517-4522.

30. Darnell Jr JE, Kerr IM \& Stark GR (1994). Jak-STAT pathways and transcriptional activation in response to IFNs and other extracellular signaling proteins. Science, 264: 1415-1421.

31. Kanno Y, Kozak CA, Schindler C, Driggers $\mathrm{PH}$, Ennist DL, Gleason SL, Darnell Jr JE \& Ozato K (1993). The genomic structure of the murine ICSBP gene reveals the presence of the $\gamma$ interferon-responsive element, to which an ISGF3 $\alpha$ subunit (or similar) molecule binds. Molecular and Cellular Biology, 13: 3951-3963.

32. Holtschke T, Löhler J, Kanno Y, Fehr T, Giese N, Rosenbauer F, Lou J, Knobelock K-P, Gabriele L, Waring J, Bachmann MF, Zinkernagel RM, Morse III HC, Ozato K \& Horak I (1996). Immunodeficiency and chronic myelogenous leukemia-like syndrome in mice with a targeted mutation of the ICSBP gene. Cell, 87: 307-317. 